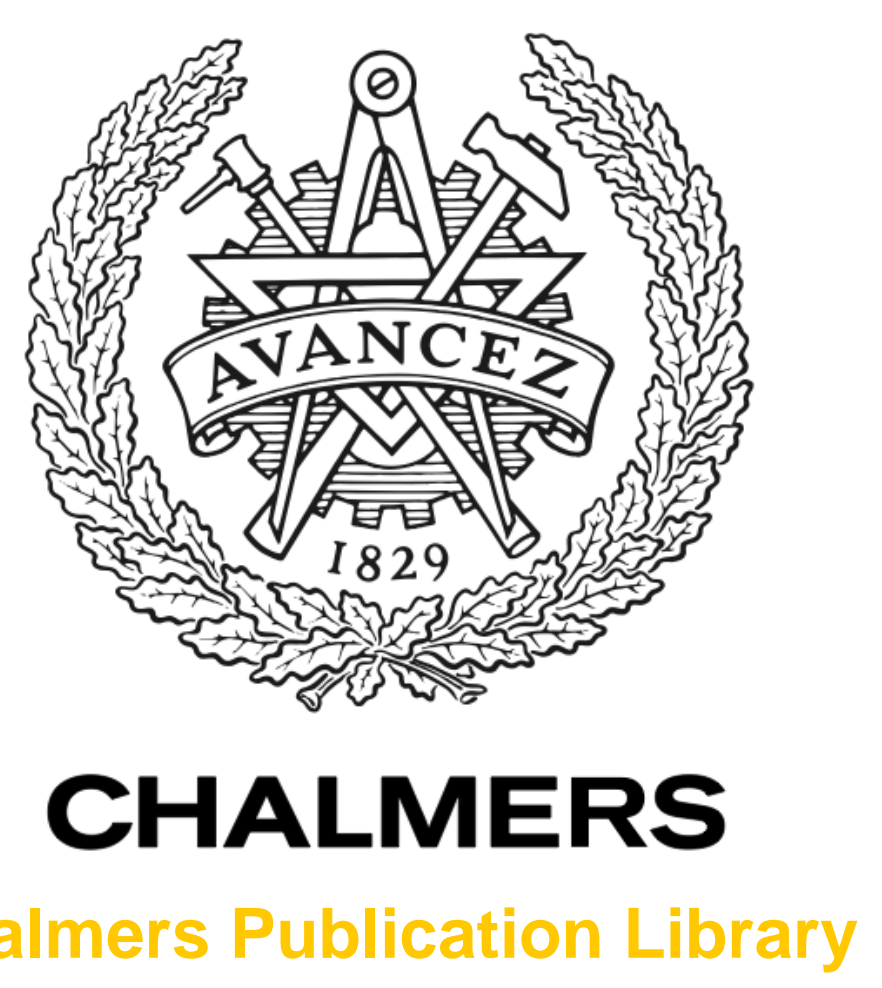

Chalmers Publication Library

\title{
Modelling of a city canyon problem in a turbulent atmosphere using an equivalent sources approach
}

This document has been downloaded from Chalmers Publication Library (CPL). It is the author's version of a work that was accepted for publication in:

Applied Acoustics (ISSN: 0003-682X)

Citation for the published paper:

Ögren, M. ; Forssén, J. (2004) "Modelling of a city canyon problem in a turbulent atmosphere using an equivalent sources approach". Applied Acoustics, vol. 65(6), pp. 629642.

http://dx.doi.org/10.1016/j.apacoust.2003.11.010

Downloaded from: http://publications.lib.chalmers.se/publication/305

Notice: Changes introduced as a result of publishing processes such as copy-editing and formatting may not be reflected in this document. For a definitive version of this work, please refer to the published source. Please note that access to the published version might require a subscription. 


\title{
Paper III
}

\section{Modelling of a city canyon problem in a turbulent atmosphere using an equivalent sources approach}

\author{
Mikael Ögren and Jens Forssén
}

\begin{abstract}
Résumé
The sound propagation into a courtyard shielded from direct exposure is predicted using an equivalent sources approach. The problem is simplified into that of a two-dimensional city canyon. A set of equivalent sources are used to couple the free half-space above the canyon to the cavity inside the canyon. Atmospheric turbulence causes an increase in the expected value of the sound pressure level compared to a homogeneous case. The level increase is estimated using a von Kármán turbulence model and the mutual coherences of all equivalent sources' contributions. For low frequencies the increase is negligible, but at $1.6 \mathrm{kHz}$ it reaches $2-5 \mathrm{~dB}$ for the geometries and turbulence parameters used here. A comparison with a ray-based model shows reasonably good agreement.
\end{abstract}

\section{Introduction}

Courtyards are shielded from direct traffic noise exposure by the surrounding buildings, and thereby they represent relatively quiet areas in urban environments. On a directly exposed façade, i.e. toward a street, the noise level can be sufficiently well predicted by standard methods based on ray-tracing (e.g. the Nordic calculation methods [1, 2]). Shielded areas seem more difficult to model. The sound paths contain multiple reflections involving diffraction, and the influence of streets further away is increased. A model for this kind of problem using equivalent sources has recently been developed for a homogeneous atmosphere [3]. Here, a further development is described, which incorporates effects of a turbulent atmosphere. The basis is a substitute sources method using a mutual coherence function for turbulence [4]. 
The situation of a depressed road, or a road surrounded by tall buildings, can be seen as a two-dimensional (2-D) problem, where the traffic will act as a line source and the road together with the buildings' façades will form a "city canyon", the sending canyon. A shielded courtyard forms a second, receiving canyon.

The equivalent sources approach to the problem is field-based rather than raybased, and thereby more easily captures the resonant behaviour of a city canyon. The original noise sources inside the sending canyon are exchanged for the equivalent sources at the top of the canyon. This can be seen as changing the position of a noise source from the canyon bottom to a typical roof height of the city, which also changes the strength and directivity of the source. The effect of turbulence is modelled on the equivalent sources on the canyon top, which is expected to be a more successful approach than using ray-based models including a scattering crosssection for turbulence. Such a scattering cross-section based method has been investigated previously and it was concluded that the turbulence influence increases at higher orders of the reflections inside the canyon [5]. This is because the higher order reflections correspond to ray directions that are more nearly horizontal, which makes the turbulence scattering stronger due to the smaller scattering angles. A precise calculation of the high order reflections together with turbulence scattering is difficult and the approach used here seems more promising.

\section{Theory}

\subsection{A 2-D canyon solution using equivalent sources}

In [6] the method of equivalent sources was used to calculate the insertion loss of balconies including absorbing surfaces. The main idea of the method is to reduce the problem to simplified geometries with boundary conditions which are easy to handle. On boundaries with different conditions, sources are placed. The strength of these sources are adjusted so that the boundary conditions are fulfilled everywhere. Applications of this can be found in $[6,7]$. The method has been shown to be robust and computationally efficient and is therefore suitable for the problem considered here.

Consider the street canyon shown in Fig. 1. In order to apply the equivalent sources method, the geometry is divided into two parts, the domain inside the canyon and the half space above $y=l_{y}$. The intersection between the two domains is denoted $C$. In this way the problem is reduced to two subproblems, which can easily be handled; radiation into a half space by a Rayleigh integral, and a sound field in a rigid cavity by a modal approach. The coupling between the half space and the 
cavity is obtained by the set of equivalent sources which correct the field impedance along the intersection. Although the mathematical derivation of the method has been described elsewhere [6], some details are repeated below for clarity.

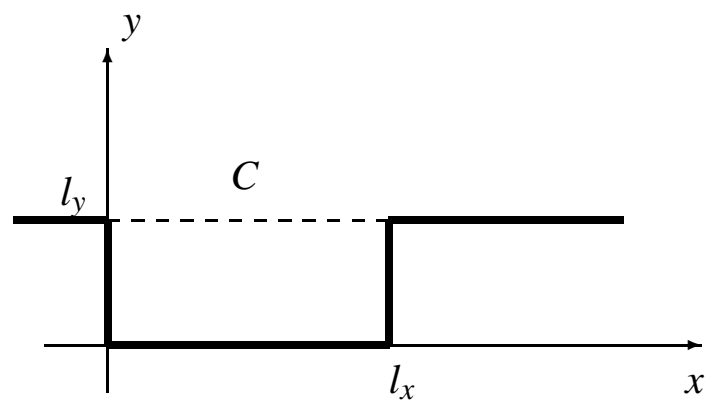

FIGURE 1 - Sketch of a two-dimensional city canyon.

In the context of boundary element methods (BEM), one can view the modal approach as finding the Green function from a velocity point source inside the canyon to the pressure at a point on the intersection $C$. Concerning numerical performance, a BEM implementation with such specialised Green functions will be equally efficient as the model presented in this paper. The rigid cavity Green functions fulfil the boundary conditions for a canyon with open top together with the equivalent sources. (If instead there had been a zero impedance boundary condition at the top, another set of Green functions is needed. For further discussion see e.g. [6].)

In the following, harmonic time dependence described by $\exp (\mathrm{j} \omega t)$ is assumed. The wave equation for the complex pressure $p$ in a two-dimensional domain, assuming a source of strength $q$, is

$$
\nabla^{2} p(x, y)+k^{2} p(x, y)=-\mathrm{j} \omega \rho_{0} q(x, y),
$$

where the strength, $q$, is a volume velocity, or in the two-dimensional case what might be called a surface velocity, and where $\rho_{0}$ is the air density. In this text $q$ denotes a distributed source, and $Q$ a point source, both in 2-D. Taking into account the boundaries of the two domains, the Green functions can be found for instance in [8] and [9], and they are

$$
G_{1}\left(x_{s}, y_{s} \mid x_{r}, y_{r}\right)=\mathrm{j} \omega \rho_{0} \frac{c^{2}}{l_{x} l_{y}} \sum_{n} \sum_{m} \frac{\Psi_{n, m}\left(x_{s}, y_{s}\right) \Psi_{n, m}\left(x_{r}, y_{r}\right)}{\Lambda_{n, m}\left(\omega_{n, m}^{2}(1+\mathrm{j} \eta)-\omega^{2}\right)},
$$


and

$$
G_{2}\left(x_{s}, y_{s} \mid x_{r}, y_{r}\right)=\mathrm{j} \omega \rho_{0} \frac{-\mathrm{j}}{2} H_{0}^{(2)}(k r) .
$$

The Green function $G_{1}$ is a modal summation where the eigen frequencies $\omega_{n, m}$ , modal shapes $\Psi_{n, m}$, and modal weights $\Lambda_{n, m}$ can be determined using

$$
\begin{aligned}
& \omega_{n, m}=\pi c \sqrt{\left(n / l_{x}\right)^{2}+\left(m / l_{y}\right)^{2}} \\
& \Psi_{n, m}(x, y)=\cos \left(n \pi x / l_{x}\right) \cos \left(m \pi y / l_{y}\right) \\
& \Lambda_{n, m}=\int_{0}^{l_{y}} \int_{0}^{l_{x}} \Psi_{n, m}^{2} \mathrm{~d} x \mathrm{~d} y,
\end{aligned}
$$

where $c$ is the sound speed, $\eta$ the loss factor, and $l_{x}$ and $l_{y}$ the dimensions of the canyon. The modal summation must be truncated somewhere, and here eigen frequencies up to three times as large as the frequency of interest were included in order to ensure convergence. This is a common truncation limit used in structural acoustics, and here it has been verified in a few test cases by increasing the number of modes and checking that the changes are small. Note that the damping expressed as $\eta$ applies to the covered canyon only, the effect of power being transferred into the field above the canyon is described by the coupling of the two domains. The damping modelling assumes that the losses are evenly distributed within the canyon. For localised areas with high damping, such as absorbers, the damping can be modelled by equivalent sources placed in these areas, fulfilling the given impedance boundary condition, as in [6].

The Green function $G_{2}$ contains the Hankel function of the second kind, and describes a line source in front of a rigid surface. The distance between the source and the receiver is $r=\sqrt{\left(x_{s}-x_{r}\right)^{2}+\left(y_{s}-y_{r}\right)^{2}}$, and $k$ is the wave number. This Green function contains no losses, but if necessary the losses due to atmospheric absorption in the propagation from the canyon to an external receiver could be included in the computations.

The loss factor $\eta$ is important for the noise level inside the canyon if the side walls of the canyon are high compared to the width. If the loss factor is determined assuming air absorption only, it will be too low. In reality the effect of finite impedances on the walls of the canyon will give higher losses. Here the loss factor is taken from reverberation time measurements at Chalmers University of Technology, in the reverberation chamber with volume $240 \mathrm{~m}^{3}$. The results showed a power-law 
behaviour and a logarithmic least squares fit of the damping as a function of frequency gives

$$
\eta(f)=10^{-0.94} f^{-0.84},
$$

valid within the frequency range of interest here. In this way the damping is underestimated, and can be seen as a kind of minimum damping for a courtyard.

The coupling between the two domains is introduced by assuming an equivalent sources distribution on the boundary. Using a combination of the primary source of strength $Q$ located inside the canyon at $\left(x_{s}, y_{s}\right)$, and the boundary source distribution $q_{l}(x)$ below the boundary and $q_{u}(x)$ above it, the pressure can be calculated as

$$
p_{l}\left(x_{r}, y_{r}\right)=Q G_{1}\left(x_{s}, y_{s} \mid x_{r}, y_{r}\right)+\int_{C} q_{l}(x) G_{1}\left(x, l_{y} \mid x_{r}, y_{r}\right) \mathrm{d} x
$$

inside the canyon and

$$
p_{u}\left(x_{r}, y_{r}\right)=\int_{C} q_{u}(x) G_{2}\left(x, l_{y} \mid x_{r}, y_{r}\right) \mathrm{d} x
$$

above the canyon. The source is assumed to be located inside the canyon for brevity.

At the intersection $C$ between the two domains, the pressure and the velocity fields must be continuous. As a consequence $p_{l}$ equals $p_{u}$ and $q_{l}$ equals $-q_{u}$ along $C$, and we can drop the subscripts $l$ and $u$. The resulting equation system can be discretised by dividing the boundary $C$ into a number of equally sized elements $C_{1}, C_{2}, \ldots, C_{N}$, and approximate the source strength along the boundary by a piecewise constant complex source strength $q_{1}, q_{2}, \ldots, q_{N}$ on each element. The pressure at the centre points of the elements, $x_{1}, x_{2}, \ldots, x_{N}$, must be equal, which gives the equation system

$$
A q=b,
$$

where

$$
A_{i, j}=\int_{C_{j}} G_{1}\left(x, l_{y} \mid x_{i}, l_{y}\right) \mathrm{d} x+\int_{C_{j}} G_{2}\left(x, l_{y} \mid x_{i}, l_{y}\right) \mathrm{d} x
$$

and

$$
b_{i}=Q G_{1}\left(x_{s}, y_{s} \mid x_{i}, l_{y}\right) .
$$

The length of the elements is set to one tenth of the wavelength. The size of the equation system will be $N \times N$, and $A$ is a symmetric matrix. Solving Eq. (10) one 
obtains the strengths of the boundary sources $q$, and can calculate the pressure anywhere inside or above the canyon using Eqs. (8) or (9), respectively.

The integrations of the free Green function $G_{2}$ in Eq. (11) can be evaluated numerically. Care has to be taken to avoid the singular part when $i=j$ (see e.g. [3]). The Green function $G_{1}$ can be integrated analytically, and the case $i=j$ does not require any special consideration.

Assuming a piecewise constant complex source distribution over each element is a rather crude approach, which can be thought of as a zeroth order polynomial approximation. Using a linear or higher order polynomial on each element might give better numerical properties, i.e. a faster and more accurate method, but this is not yet studied further.

\subsection{Modelling of turbulence effects}

In the general case with contributions from two sources to one receiver, the two paths can have a transversal separation as well as different lengths. Here, all the paths follow a single line, along the intersection $C$, and only the lengths vary. The mutual coherences for such cases can be estimated using the extinction coefficient, $\gamma$, for a turbulent atmosphere.

Here, we assume that the turbulence is homogeneous and isotropic, i.e. has statistical properties independent of translation and rotation. This is a crude approximation; in reality we expect the canyons to affect the turbulence, in addition to the variations with height one gets over any surface. Such refinements should however be possible to include in the model. Yet another approximation is used, which is that there is no turbulence inside the canyon. It might be possible to extended the model to incorporate turbulence inside the canyon. As a result we would expect a larger effect of turbulence.

The ensemble average of the pressure amplitude, $\langle p\rangle$, decays exponentially as the wave propagates through a turbulent medium. This can be formulated as

$$
\langle p\rangle=\hat{p} \mathrm{e}^{-\gamma x},
$$

where $\hat{p}$ is the amplitude in absence of turbulence and $x$ is the distance of propagation [10]. The average pressure, $\langle p\rangle$, is also called the coherent field.

If the path from one source to the receiver is extended a distance $\Delta$ compared to the path from the other source, the mutual coherence factor of the two contributions is estimated as $\mathrm{e}^{-\gamma \Delta}$, as explained next. The definition used here of the mutual 
coherence factor for two contributions is

$$
\Gamma_{12}=\frac{\left\langle p_{1} p_{2}^{*}\right\rangle+\left\langle p_{1}^{*} p_{2}\right\rangle}{\hat{p}_{1} \hat{p}_{2}^{*}+\hat{p}_{1}^{*} \hat{p}_{2}}
$$

where the complex conjugate is denoted by an asterisk $\left(^{*}\right), p_{1}$ and $p_{2}$ are the fluctuating pressure amplitudes in the turbulent atmosphere and $\hat{p}_{1}$ and $\hat{p}_{2}$ are the amplitudes for the same situation except that there is no turbulence (e.g. [11]). The propagation for $p_{1}$ and $p_{2}$ follow the same paths all the way except along the extension with length $\Delta$. Only the propagation along the extension causes decorrelation (i.e. reduced coherence) since the rest of the propagation goes through the same medium for both paths. Using Eq. (13) this results in $\left\langle p_{1} p_{2}^{*}\right\rangle=\hat{p}_{1} \hat{p}_{2}^{*} \mathrm{e}^{-\gamma \Delta}$ and $\left\langle p_{1}^{*} p_{2}\right\rangle=\hat{p}_{1}^{*} \hat{p}_{2} \mathrm{e}^{-\gamma \Delta}$, which gives $\Gamma_{12}=\mathrm{e}^{-\gamma \Delta}$. This estimate of the mutual coherence is equivalent to assuming turbulence only along the intersection above the canyon, and not further away. In a more accurate solution of the received pressure on the same line as the two sources, not only the propagation along the extension between the sources should affect the decorrelation if turbulence is present all the way to the receiver. In terms of Fresnel zones, the field from the source further away from the receiver will cover a larger volume of the atmosphere and thereby be more affected by turbulence. Hence, the model used here is assumed to underestimate the effect of turbulence.

Each equivalent source of the sending canyon gives a contribution $p_{i}, i=1, \ldots, N$, to the received pressure. The total contribution including all mutual coherences can be written (e.g. [12])

$$
\left\langle\left|p_{\text {tot }}\right|^{2}\right\rangle=\sum_{i} \sum_{j}\left\langle p_{i} p_{j}^{*}\right\rangle=\sum_{i} \sum_{j} \hat{p}_{i} \hat{p}_{j}^{*} \Gamma_{i j}
$$

where $\Gamma_{i j}=\mathrm{e}^{-\gamma \Delta_{i j}}$, with $\Delta_{i j}$ the distance between the equivalent sources $i$ and $j$. Eq. (15) gives the expected value of the square of the pressure amplitude in the turbulent medium, and is used to estimate the equivalent level.

In a situation with two canyons (see Fig. 2), a sending and a receiving one, it can be shown that the decorrelation due to turbulence can be treated separately for the two canyons. For this, however, a far field condition needs to be fulfilled, i.e. that the widths of the canyons are small in comparison to the distance in between them. This results in an excitation from the sending canyon at the boundary, $C_{I I}$, of the receiving canyon. The excitation has constant amplitude, $Q_{1}$, and phase variation $\exp (-\mathrm{j} k x)$ along $C_{I I}$. The amplitude is found from $Q_{1}^{2}=\sum_{i} \sum_{j} q_{i} q_{j}^{*} \Gamma_{i j}$, where $q_{i}$ and $q_{j}$ are the contributions via the equivalent sources $i$ and $j$ on $C_{I}$, with coherence $\Gamma_{i j}$. 


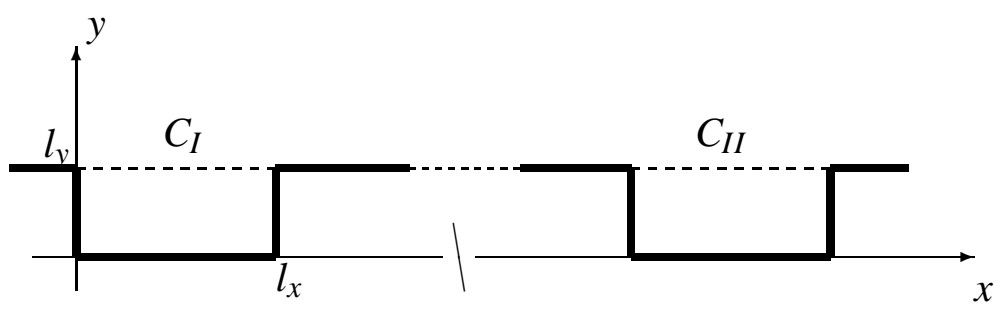

FIGURE 2 - Sketch of two city canyons.

The contributions via the different equivalent sources at $C_{I I}$ give rise to a similar double sum and the total result can be written

$$
\left\langle\left|p_{\text {tot }}\right|^{2}\right\rangle=Q_{1}^{2} \sum_{i} \sum_{j} g_{i} g_{j}^{*} \Gamma_{i j},
$$

where $g_{i}$ and $g_{i}$ are the Green functions, in absence of turbulence, from $Q_{1}$ to the received pressure inside the canyon via the equivalent sources $i$ and $j$ at $C_{I I}$, with coherence $\Gamma_{i j}$. It could be noted that the Green functions in Eq. (16) are numerically found from the reciprocal problem, where the source position (i.e. where $Q_{1}$ was taken) and the receiver position are interchanged.

For the calculated results shown here, the von Kármán turbulence model is used, for which the extinction coefficient can be written [10]

$$
\gamma=\gamma_{T}+\gamma_{v}=\frac{3}{10} \pi^{2} A k^{2} K_{0}^{-5 / 3}\left(\frac{C_{T}^{2}}{T_{0}^{2}}+\frac{4 C_{v}^{2}}{c^{2}}\right) .
$$

In the above equation $\gamma_{T}$ and $\gamma_{v}$ are the extinction coefficients due to temperature and velocity fluctuations, respectively; $A \approx 0.0330 ; K_{0}=2 \pi / L_{0}$, where $L_{0}$ approximates the outer scale of turbulence; $C_{T}^{2}$ and $C_{v}^{2}$ are the structure parameters describing the strengths of temperature and velocity fluctuations, respectively; $T_{0}$ is the mean temperature; and $c$ is the mean sound speed.

\section{Results}

In the calculations, the canyons modelled are $18 \mathrm{~m}$ high and 19 or $11 \mathrm{~m}$ wide. All surfaces are acoustically hard. The source is on the bottom of the canyon, at position $x_{s}=9$ or $5 \mathrm{~m}$, for the 19 and $11 \mathrm{~m}$ wide canyon, respectively. (These data 
are summarised in Table 1.) For the single-canyon problems, the receiver is placed at $x=500 \mathrm{~m}$, on the hard surface. The results apply equally well to the reciprocal problem, with the receiver in the canyon and the source $500 \mathrm{~m}$ away. For the doublecanyon problems, the receiving canyon starts at $x=500 \mathrm{~m}$. All results are plotted relative to free field.

\begin{tabular}{cccc} 
Canyon & Width $l_{x} \mathrm{~m}$ & Height $l_{y} \mathrm{~m}$ & Source/receiver pos. \\
\hline $\mathrm{A}$ & 19 & 18 & $(9,0)$ \\
$\mathrm{B}$ & 11 & 18 & $(5,0)$
\end{tabular}

TABLE 1 - Canyon geometries used for the calculations. The source/receiver positions are slightly off center in order to get contribution from modes with both odd and even orders in the $x$ direction.

The double-canyon problem models a sending canyon (road) and a receiving canyon (closed courtyard). The calculations are then divided into two steps. First the sending canyon is treated as if the receiving canyon is not present. Then the strengths of the equivalent sources calculated in the first step are seen as sources on a rigid plane for the receiving canyon, and the pressure at the receiving point is calculated. This approach is valid as long as the waves that are reflected at the receiving canyon back to the source canyon, and then back again to the receiving canyon, can be neglected.

For the turbulence modelling we have used velocity fluctuations with $C_{v}^{2}=$ $10 \mathrm{~m}^{4 / 3} \mathrm{~s}^{-2}$ and $L_{0}=10 \mathrm{~m}$. The value of $C_{v}^{2}$ is taken from measurements and chosen to model a strong turbulence condition [13]. The size of the largest scales of influence, $L_{0}$, can in general be much larger [14] but here propagation fairly close to ground is modelled and a smaller value is chosen, which results in a smaller value of the extinction coefficient and in a weaker turbulence influence. For these values of the turbulence parameters, examples of the mutual coherence factor, $\Gamma$, (see Eqs. 13 and 17) are plotted in Fig. 3 for different sound frequencies. The value of $\Gamma$ is 1 at separation $x=0$ and decays for larger absolute values of $x$.

The main results presented here are third octave band levels, each calculated using 20 frequencies, starting at $100 \mathrm{~Hz}$ and ending at $1.6 \mathrm{kHz}$. However, in Section 3.1 below the results are calculated for a higher resolution in frequency, near $500 \mathrm{~Hz}$.

\subsection{Comparison with a ray-based model}

In Fig. 4 two different methods for estimating the influence of turbulence for a single $19 \mathrm{~m}$ wide canyon is presented. The upper figure is calculated using the 


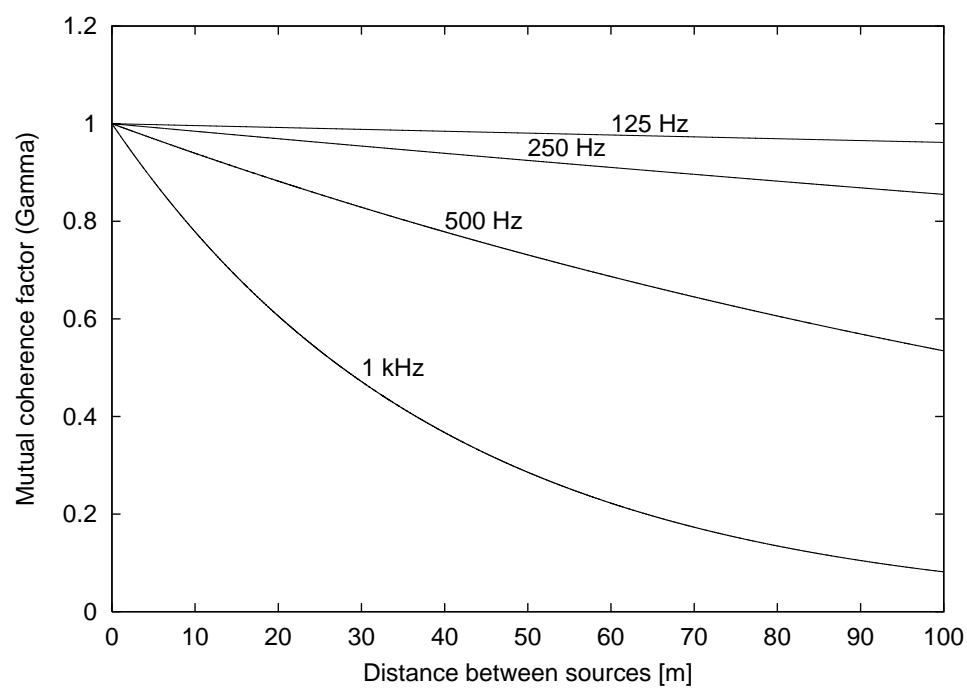

FIGURE 3 - Calculation of the mutual coherence factor $\Gamma=\mathrm{e}^{-\gamma x}$ versus distance, $x$, for different frequencies.

equivalent sources method as described in section 2.1, and the lower is calculated using a ray-based model, where the diffraction and reflection modelling is described in [15]. The diffraction theory is combined with the concept of Fresnel zones to reduce the strength of reflections from the finite, vertical surfaces. Here, a parameter value of $1 / 8$ of a wavelength is used for the Fresnel zone approach, as recommended in the new Nordic sound propagation method for finite reflecting surfaces [2]. A maximum of 32 reflections are taken into account, and numerical tests showed small increase when including more reflections.

The turbulence effect is estimated using the simplified scheme described in [13], which is based on a scattering cross-section for a turbulent atmosphere. Here the turbulence parameters together with geometry parameters such as the distance to and the height of the screening object is used to determine the scattered level. This level is then added incoherently to the diffracted level to produce the expected value of the total level behind the screen. This combination of reflections, diffractions and turbulence scattering has previously been used to estimate the influence of multiple reflections and distant sources in city environments [5].

For the equivalent sources approach (upper plot in Fig. 4), the turbulence can be seen to have two effects, working in opposite directions. First, the decorrelation 

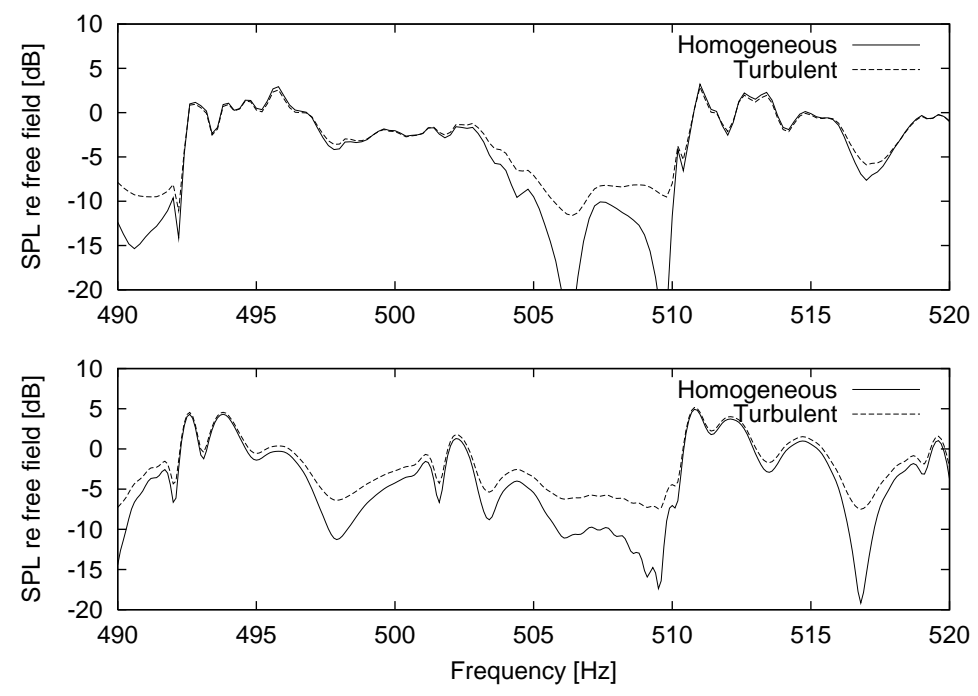

FIGURE 4 - Frequency response relative free field for a canyon of width $19 \mathrm{~m}$ and height $18 \mathrm{~m}(\mathrm{~A})$. The receiver is outside the canyon at $(500,18)$. The upper figure is calculated using the equivalent sources method, and the lower using the ray-based model.

weakens the positive interference slightly, as can be seen at the largest resonance peaks, which leads to a decreased level. Second, the strongest shadowing, in between the resonance peaks, is limited, which leads to an increased level. This can be seen as a weakened destructive interference of the equivalent sources' contributions.

For the ray-based model only the second effect is visible; since the scattered level is added incoherently, it can only lead to increased levels. The total effect for both approaches, averaged over several resonances, is however an increase due to the turbulence. In the case presented here the increase averaged over the third octave bands $1,1.25$ and $1.6 \mathrm{kHz}$ is $1.6 \mathrm{~dB}$ for the equivalent sources method and $4.9 \mathrm{~dB}$ for the ray-based model.

In narrow bands the results from the two methods show the same trends at peaks and dips, but are far from perfectly matched. It should be stressed that the ray-based model has significant weaknesses. For instance, the Fresnel zone approach can be implemented with different parameters, and the diffraction theory has limitations at low frequencies and high diffraction orders. On the other hand, the equivalent sources method without the turbulence modelling has been validated against BEM, 
with good agreement [3], and is concluded to be more accurate than the ray-based model.

\subsection{Results for single and double canyons}

In Figs. 5 and 6 third octave band results are shown for the 19 and $11 \mathrm{~m}$ wide canyon, respectively (canyons A and B in Table 1). It can be seen that the increase in sound level due to turbulence is larger for the wider canyon (Fig. 5), where the strongest decorrelation takes place.

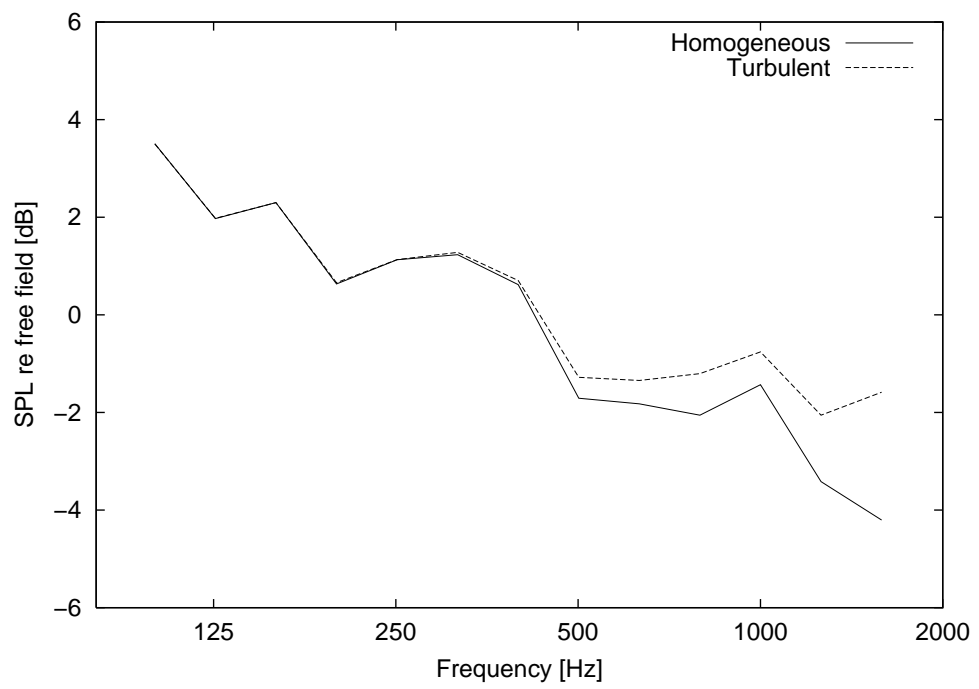

FIGURE 5 - Frequency response in third octave bands relative free field for a canyon of width $19 \mathrm{~m}$ and height $18 \mathrm{~m}$ (A). The source is located at $(9,0)$ and the receiver at $(500,18)$.

Figs. 7-9 are for double-canyon problems. The receiver is at the bottom of a 19 or $11 \mathrm{~m}$ wide canyon, at a distance of 9 or $5 \mathrm{~m}$ from the wall closest to the sending canyon, respectively. Fig. 7 is for a sending canyon of $19 \mathrm{~m}$ width and a receiving canyon of 19 or $11 \mathrm{~m}$ width. The turbulence decorrelation is only modelled for the sending canyon and the calculations at each frequency should give the same increase due to turbulence since the width of the canyon with turbulence is the same. The effect on the third octave band levels in the receiving canyon is however not the same; the receiving canyon can be seen as filtering the input from the sending 


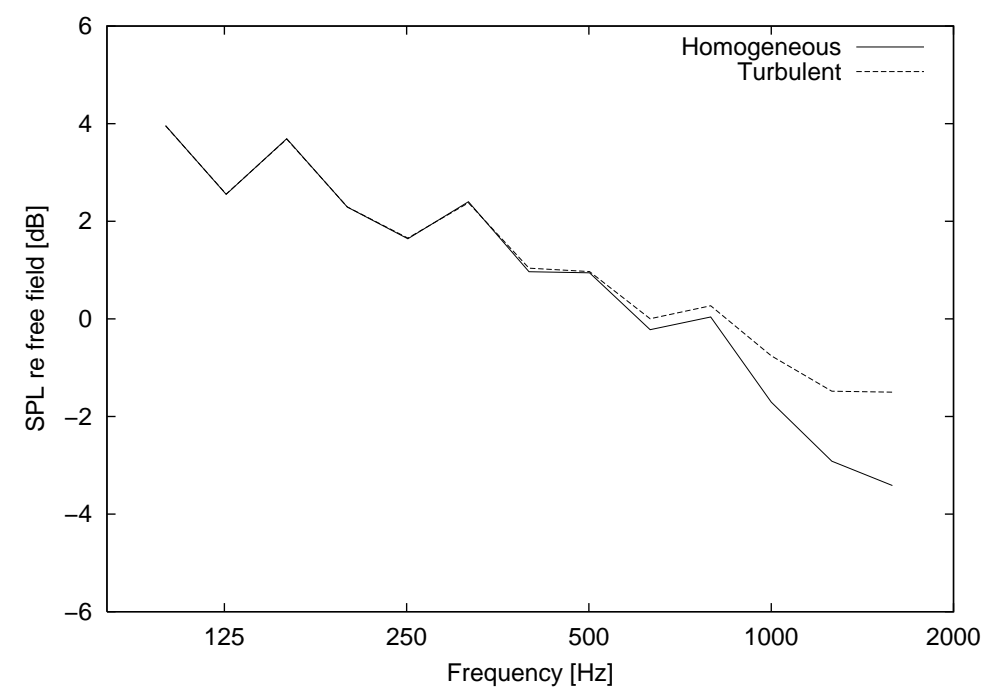

FIGURE 6 - Frequency response in third octave bands relative free field for a canyon of width $11 \mathrm{~m}$ and height $18 \mathrm{~m}(\mathrm{~B})$. The source is located at $(5,0)$ and the receiver at $(500,18)$.

canyon before the third octave band levels are calculated. For two canyons of equal width, the turbulence caused level increase in between the resonance peaks of the sending canyon gets a reduced influence when the receiving canyon has the same resonance behaviour. We can see in Fig. 7 that the $11 \mathrm{~m}$ wide receiver canyon (B) gives a larger influence of turbulence.

Fig. 8 shows the results for two situations with turbulence at the sending canyon. The first situation has a $19 \mathrm{~m}$ wide sending canyon and an $11 \mathrm{~m}$ wide receiving canyon (A to B), whereas the second situation has the widths interchanged (B to A). The influence of the turbulence on the field from the sending canyon is filtered by the receiving canyon. Thereby the trend in the results that a wider canyon should be more sensitive to turbulence is less distinguished.

The results from modelling turbulence in both canyons (A and B) are shown in Fig. 9. The separate treatment of the turbulence effects here means that the increase would equal the sum of the increases in the two cases shown in Fig. 8 if single-frequency results were shown. The third octave band averaging can change this slightly, but we expect to get larger influence of turbulence when it is modelled in both canyons than in only one canyon. Here, the effect is more than $5 \mathrm{~dB}$ at 
$1.6 \mathrm{kHz}$.

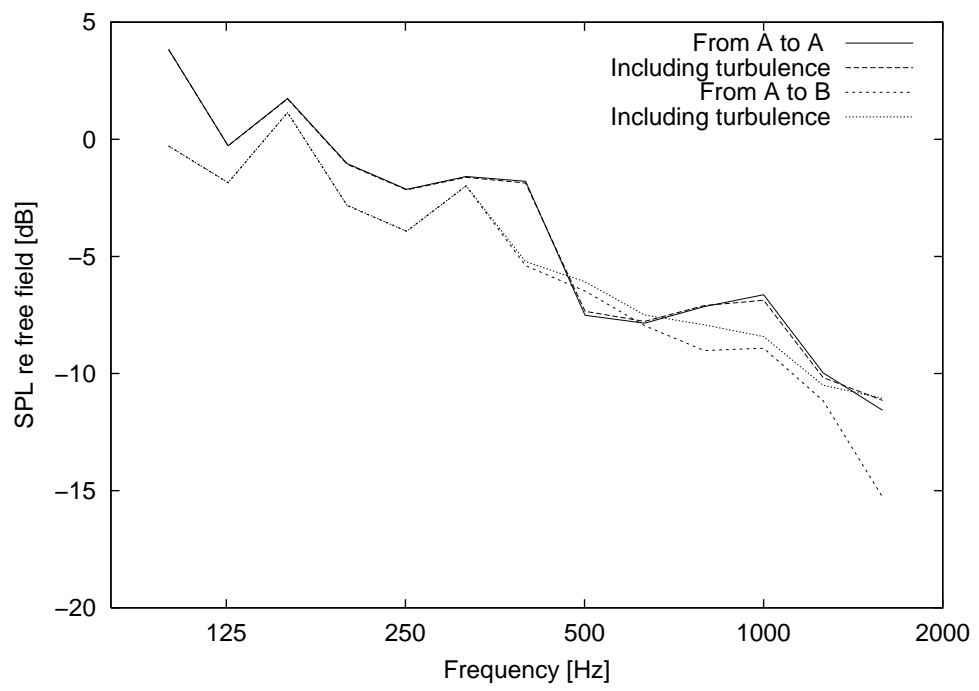

FIGURE 7 - Frequency response in third octave bands relative free field for a doublecanyon situation. The effect of turbulence is included for the source canyon only, which is $19 \mathrm{~m}$ wide (B).

\section{Conclusions}

The increase in the sound pressure level due to turbulence can be predicted for city canyons. The model is based on the mutual coherence factor for sources or receivers separated in space in a turbulent atmosphere, and assumes a homogeneous and isotropic turbulence described by the von Kármán model.

The equivalent sources approach to the problem is expected to more easily capture the resonant behaviour of a city canyon than a ray-based model. The original noise sources inside the canyon can be seen as being lifted up to the roof level of the city when replaced by the equivalent sources. The effect of turbulence is modelled on the equivalent sources using a mutual coherence factor, which is thought to be a more successful method than a ray-based one using a scattering cross-section instead.

The level increase due to turbulence is negligible at low frequencies but starts to become important around $500 \mathrm{~Hz}$ with the geometries and parameters used here. 


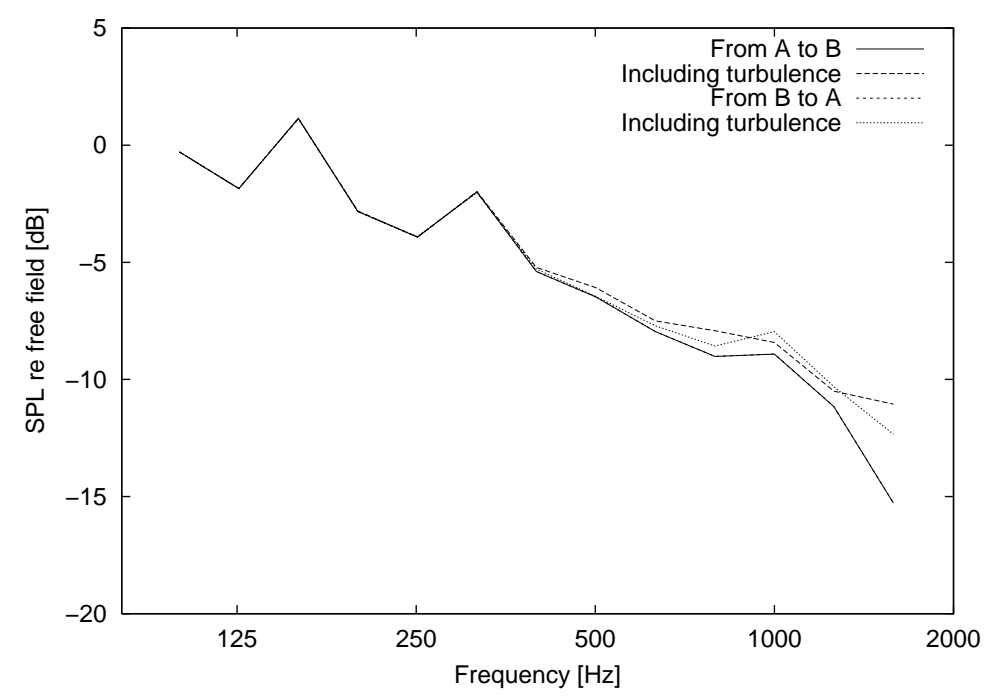

FIGURE 8 - Frequency response in third octave bands relative free field for a doublecanyon situation. The effect of turbulence is included for the source canyon only, which is $19 \mathrm{~m}$ or $11 \mathrm{~m}$ wide (A or B). (Without turbulence the results are identical for $\mathrm{A}$ to $\mathrm{B}$ and $\mathrm{B}$ to $\mathrm{A}$.)

At the third octave band $1.6 \mathrm{kHz}$ the increase reaches $2-5 \mathrm{~dB}$. Using a traffic noise spectrum $\left(C_{t r}\right.$ in [16]) to estimate the effect in the A-weighted level gives slightly less than $1 \mathrm{~dB}$ increase compared to the homogeneous case. These calculated values do however depend on the geometry and turbulence parameters. For larger geometries and stronger turbulence the effect of turbulence is expected to increase.

For future improvements of predictions in canyon-to-canyon cases it is important to include refraction, at least for canyons that are far from each other. More realistic damping data are also needed, either from measurements on real courtyards or indirectly from measurements on typical façade materials. It is also difficult to know what values to use for the turbulence parameters. Hopefully more input from research in the field of urban micro climate can help.

\section{Acknowledgements}

This paper is based on a study performed within the research programme "Soundscape Support to Health", sponsored by the Swedish Foundation for Strategic Envi- 


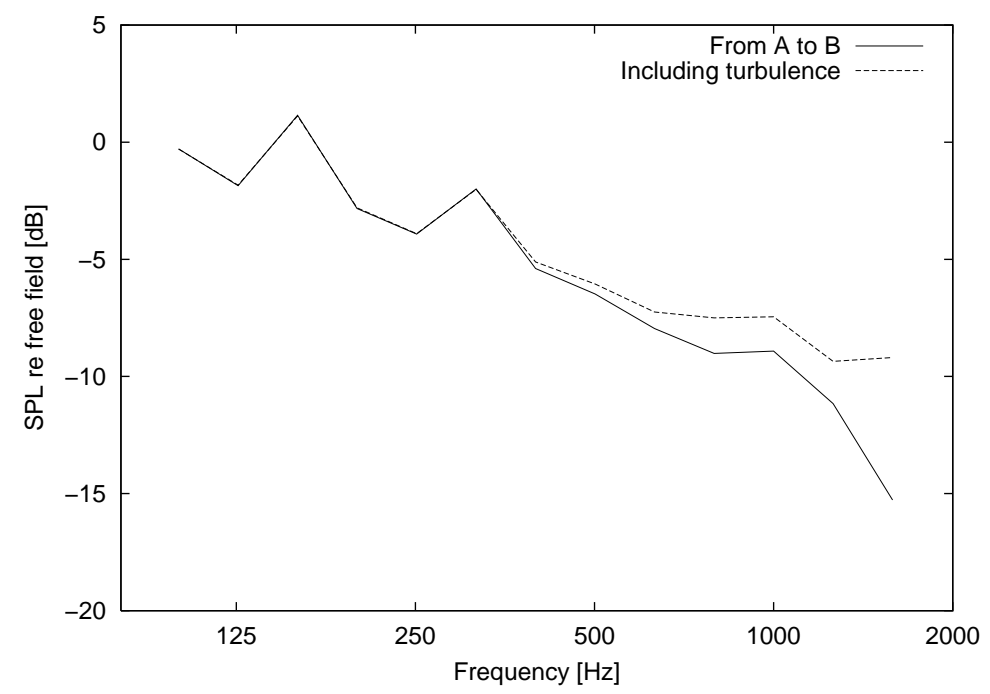

FIGURE 9 - Frequency response in third octave bands relative free field for a doublecanyon situation. The effect of turbulence is included for both canyons (A and B).

ronmental Research (MISTRA), the Swedish Agency for Innovation Systems (Vinnova) and the Swedish National Road Administration (VV). The work behind this paper has also been funded by Formas (the Swedish Research Council for Environment, Agricultural Sciences and Spatial Planning).

\section{Références}

[1] Jonasson H, Nielsen H. Road traffic noise - nordic prediction method. TemaNord 1996 :525, Copenhagen, Nordic Council of Ministers, 1996.

[2] Plovsing B, Kragh J. Nord2000. Comprehensive Outdoor Sound Propagation Model. Part 1 : Propagation in an Atmosphere without Significant Refraction. Delta report AV 1849/00, Lyngy, Denmark, 2000.

[3] Ögren M, Kropp W. Road traffic noise propagation between two-dimensional city canyons using an equivalent sources approach. Submitted to AcusticaActa acustica, March 2003. 
[4] Forssén J. Calculation of noise barrier performance in a turbulent atmosphere by using substitute sources above the barrier. Acustica - Acta acustica $2000 ; 86: 269-75$.

[5] Ögren M, Forssén J. Prediction of noise levels in shielded urban areas. Proc. Internoise 2001, The Hague 2001.

[6] Bérillon J, Kropp W. A theoretical model to consider the influence of absorbing surfaces inside the cavity of balconies. Acustica - Acta acustica $2000 ; 86$ :485494.

[7] Cummings A. The effects of a resonator array on the sound field in a cavity. Journal of Sound and Vibration $1992 ; 154(1): 25-44$.

[8] Kuttruff H. Room acoustics. 2 ed. London, Applied science publishers Ltd., 1979.

[9] Skudrzyk E. The foundations of acoustics. New York - Wien, Springer - Verlag, 1971.

[10] Ostashev VE. Acoustics in moving inhomogeneous media. London, E \& FN Spon (an imprint of Thomson Professional), 1997.

[11] Salomons, EM. The fluctuating field of a monopole source in a turbulent atmosphere above a ground surface. Time-averaged sound pressure level and statistical distributions. Proc. 8th Int. Symp. on Long-Range Sound Propagation, The Pennsylvania State University 1998 :326-351.

[12] L'Espérence A, Herzog P, Daigle GA, and Nicolas, JR. Heuristic model for outdoor sound propagation based on an extension of the geometrical ray theory in the case of a linear sound speed profile. Applied Acoustics $1992 ; 37: 111$ 139.

[13] Forssén J, Ögren M. Barrier noise-reduction in the presence of atmospheric turbulence : Measurements and numerical modelling. Applied Acoustics $2001 ; 63: 173-187$.

[14] Wilson KD. A turbulence spectral model for sound propagation in the atmosphere that incorporates shear and buoyancy forcings. Journal of the Acoustical Society of America $2000 ; 108$ :2021-38.

[15] Salomons E. Sound propagation in complex outdoor situations with a nonrefracting atmosphere : Model based on analytical solutions for diffraction and reflection. Acustica - Acta acustica $1997 ; 83: 436-454$.

[16] Acoustics - rating of sound insulation in buildings and of building elements part 1 : Airborne sound insulation. ISO 717-1 :1996, The International Organization for Standardization, 1996. 